\title{
Co-Occurring Polysubstance Use and Physical Disease of Persons with Mental Disorders in Residential Treatment Program
}

\author{
Minjeong Kim¹, Linda Chafetz ${ }^{2}$ \\ ${ }^{1}$ School of Nursing, San Diego State University, San Diego, USA \\ ${ }^{2}$ School of Nursing, University of California, San Francisco, USA \\ Email: minjeong.kim@sdsu.edu
}

How to cite this paper: Kim, M. and Chafetz, L. (2020) Co-Occurring Polysubstance Use and Physical Disease of Persons with Mental Disorders in Residential Treatment Program. Open Journal of Nursing, 10, 1186-1194.

https://doi.org/10.4236/ojn.2020.1012085

Received: January 24, 2020

Accepted: December 15, 2020

Published: December 18, 2020

Copyright $\odot 2020$ by author(s) and Scientific Research Publishing Inc. This work is licensed under the Creative Commons Attribution International License (CC BY 4.0).

http://creativecommons.org/licenses/by/4.0/

\begin{abstract}
The purpose of this study was to describe the prevalence of co-occurring polysubstance use and to examine the differences in physical disease between polysubstance users and single/no substance users with mental disorders. This retrospective cross-sectional study included 1949 clinical records of psychiatric patients from 11 residential treatment programs between 2007 and 2011. Demographic variables, psychiatric diagnoses, and data on substance use and physical disease were obtained from the clinical records. Chi-square analyses were used to examine substance use difference in the prevalence of each physical disease category. This study found that the prevalence of co-occurring polysubstance use was $53.5 \%$. Chi-square analyses identified that co-occurring polysubstance users reported more respiratory, digestive, musculoskeletal, and HIV/AIDS diseases but less endocrine diseases than single/no substance users. Therefore, integrated treatment programs for treating patients with co-occurring substance use and physical disease should be developed and expanded for this high-risk group.
\end{abstract}

\section{Keywords}

Mental Disorders, Physical Disease, Polysubstance Use

\section{Introduction}

Current research studies have explored relationships among mental disorder, substance use, and physical disease. Persons with mental disorders compared to those without mental disorders reported higher prevalence in binge drinking ( $31 \%$ vs. $25 \%)$ and illicit drug use (37\% vs. $16 \%)$. This past-year's preva- 
lence of co-occurring substance use and mental disorder was 3.7\% [1] and lifetime prevalence was about $7 \%$ based on another study [2]. Persons who used substances such as cocaine, cannabis, opiates, and amphetamines were more likely to report psychotic symptoms compared to those who did not use substances [3]. However, among Medicaid beneficiaries, there was no significant difference in the prevalence of substance use between persons with mental disorders and those without mental disorders [4]. Although there were some inconsistent findings in the prevalence of co-occurring substance use and mental disorder, it is generally considered that mental disorder is a risk factor for substance use.

In terms of physical disease, Medicaid beneficiaries with mental disorders reported a significantly higher prevalence of physical diseases such as hypertension, heart disease, diabetes, gastrointestinal disorders, skin infections, malignant neoplasm, asthma, and acute respiratory disorders than those without mental disorders. Also, persons with mental disorders showed a higher prevalence in having two or more physical diseases than those without mental disorders ( $26 \%$ vs. $12 \%)$ [4]. In another study, about $20 \%$ of the psychiatric patients reported substance use and showed higher prevalence in infectious and digestive diseases. Co-occurring substance users, however, showed a lower prevalence in endocrine disease [5]. Based on the literature, having mental disorders with or without substance use may lead to an increased risk of certain types of and numbers of physical disease.

Although knowledge about the relationships among mental disorder, substance use, and physical disease has been established, little is known about co-occurring polysubstance use and physical disease in persons with mental disorders. Polysubstance use refers to the concurrent or simultaneous use of two or more substances including illicit drugs or non-medically used prescription-type drugs with a dependence upon at least one substance [6]. Polysubstance use is common among substance users. In publically funded treatment programs, 56\% of the users were admitted for treatment of their polysubstance use [7]. The most common pattern of polysubstance use was the combination of alcohol and other drugs. About $12 \%$ of the polysubstance users reported a combination of alcohol and cocaine, followed by $8.9 \%$ reporting a combination of alcohol, cocaine, and marijuana, and 7.4\% reporting a combination of alcohol and marijuana [8]. Co-occurring polysubstance users may have an increased risk of physical disease, thus needing more intensive treatment and care than single/no substance users. Therefore, there is a need to look at substance use differences in physical diseases in persons with mental disorders.

The purposes of this study were to 1) describe the prevalence of co-occurring polysubstance use in patients with mental disorders in residential treatment programs; and 2) examine differences in the prevalence of physical disease between co-occurring polysubstance users and single/no substance users in residential treatment programs. 


\section{Methods}

\subsection{Study Design}

This study used a retrospective cross-sectional design to look at the prevalence of co-occurring polysubstance use and physical disease in residential treatment program users with mental disorders. It used clinical data obtained from residential treatment programs in California. This data was from the Primary Health Care for the Mentally Ill Adults study (Principal Investigator: Dr. Chafetz). This study was approved by the Committee on Human Research, University of California San Francisco (UCSF).

\subsection{Sample and Setting}

This study included 1949 adult patients with mental disorders in the San Francisco Bay Area, California. This study was conducted between 2011 and 2013. Inclusion criteria were: 1) being at least 18 years old; 2) having at least one psychiatric disorder; 3 ) ever having used residential treatment services provided by the UCSF School of Nursing's clinical faculty between 2007 and 2011; and 4) having a recent clinical record if one had received services more than one time. Exclusion criteria were: 1) being under 18 years old; 2) never having been diagnosed with a psychiatric disorder; 3) never having used residential treatment services provided by the UCSF School of Nursing's clinical faculty between 2007 and 2011; and 4) not having a recent clinical record if one had received the services more than once.

The settings were 11 residential treatment programs including crisis, transitional, and long-term treatment programs, operated by the San Francisco Progress Foundation. Individuals who are referred from inpatient or crisis services are provided with voluntary care in these home-like programs. As a faculty community outreach practice, the clinical faculty at the UCSF School of Nursing provide integrated primary health care to the residential treatment program users.

\subsection{Data Collection}

Data were extracted from clinical records by Nurse Practitioners (NPs). Demographic variables included age; gender (male vs. female); race/ethnicity (European American, African American, Mexican American, and others); living condition (homeless vs. other housing); insurance status (insured vs. uninsured); and smoking status (current smoker vs. non-smoker). Initially, up to three psychiatric diagnoses were extracted from the clinical records, and then the principal psychiatric diagnosis, which was the main reason for the referral to these residential treatment programs, was used for this study. Classifications based on the Diagnostic and Statistical Manual-IV (DSM-IV) included schizophrenia; schizoaffective disorder; bipolar disorder; depressive disorder; anxiety disorder; psychosis not other specified (PNOS); and other psychiatric disorders (i.e. eating disorders, etc.) [9]. Substance use was noted as yes/no for current use of alcohol, stimulants, marijuana, opiate, sedative/hypnotics, and hallucinogens, and then 
dichotomized into polysubstance use vs. single/no substance use. All medical diagnoses or chief complaints were initially taken from the clinical records, and then categorized into one of the major six disease categories based on the international classification of diseases-9 (ICD-9): circulatory disease (hypertension and other cardiac diseases); respiratory disease (asthma, emphysema, chronic obstructive pulmonary disease (COPD), bronchitis, and upper respiratory infection (URI)); digestive disease (gastrointestinal conditions and liver disease); endocrine disease (diabetes and thyroid disease); musculoskeletal disease (joint disease and injuries); and HIV/AIDS infection [10].

\subsection{Data Analyses}

The SPSS 21 was used to analyze data in this study. In order to explore sample characteristics, descriptive statistics were used including mean $(M)$ and standard deviations $(S D)$ for age and frequencies and percentage for all categorical variables. Participants with two or more substances were categorized as polysubstance users and those with at least one substance as single/no substance users. In order to examine substance use differences in the prevalence of physical disease, Chi-square tests were analyzed for each physical disease between polysubstance users and single/no substance users. Significance level was set at an alpha level of 0.05 .

\section{Results}

The demographic characteristics of the participants are shown in Table 1. The mean age of the participants was 41.6 years $(S D=11.6)$. About $67 \%(n=1314)$ of the participants were males and about $33 \%(n=635)$ were females. Of the racial/ethnic groups, about $46 \%(n=900)$ of the participants were European American, followed by $28.5 \%$ African American $(n=555), 13.2 \%$ other racial/ethnic groups $(n=257)$, and $12.1 \%$ Mexican American $(n=237)$. About $62 \%$ of the participants were homeless and insured. Most of the participants were current smokers $(n=1459,80.0 \%)$. For the principal psychiatric disease, about $40 \%(n=781)$ of the participants had depressive disorder, followed by $13.1 \%$ bipolar disorder $(n=255), 11.6 \%$ PNOS $(n=227), 11.0 \%$ schizophrenia ( $n=214), 10.9 \%$ other minor psychiatric disorders $(n=212), 9.1 \%$ schizoaffective disorder $(n=178)$, and $4.2 \%$ anxiety disorder $(n=82)$.

Table 2 shows the patterns of substance use among the study's participants. Among substances, alcohol was the most commonly used substance $(n=1204$, $61.8 \%$ ), followed by stimulants ( $n=1000,51.3 \%)$, marijuana $(n=626,32.1 \%)$, opiates $(n=358,18.4 \%)$, sedatives/hypnotics $(n=175,9.0 \%)$, and hallucinogens $(n=125,6.4 \%)$. More than half of the participants $(n=1042,53.5 \%)$ were identified as polysubstance users and $46.5 \%(n=907)$ as single/no substance users.

Prevalence of physical disease is shown in Table 3. Circulatory disease $(n=$ $459,24.0 \%)$ was the most frequently reported disease, followed by digestive disease $(n=445,22.8 \%)$, musculoskeletal disease $(n=364,17.4 \%)$, respiratory 
Table 1. Demographic characteristics of the participants $(n=1949)$.

\begin{tabular}{|c|c|c|}
\hline Variable & $n / M$ & $\% / S D$ \\
\hline Age & 41.6 & 11.6 \\
\hline \multicolumn{3}{|l|}{ Gender } \\
\hline Male & 1314 & 67.4 \\
\hline Female & 635 & 32.6 \\
\hline \multicolumn{3}{|l|}{ Race/Ethnicity } \\
\hline European American & 900 & 46.2 \\
\hline African American & 555 & 28.5 \\
\hline Mexican American & 237 & 12.1 \\
\hline Others & 257 & 13.2 \\
\hline \multicolumn{3}{|l|}{ Living condition } \\
\hline Homeless & 1213 & 62.2 \\
\hline Others & 736 & 37.8 \\
\hline \multicolumn{3}{|l|}{ Insurance } \\
\hline Insured & $1136^{*}$ & 62.6 \\
\hline Uninsured & $679^{*}$ & 37.4 \\
\hline \multicolumn{3}{|l|}{ Smoking status } \\
\hline Smoker & $1459^{*}$ & 80.0 \\
\hline Non-smoker & $365^{*}$ & 20.0 \\
\hline \multicolumn{3}{|l|}{ Principal psychiatric disorder } \\
\hline Schizophrenia & 214 & 11.0 \\
\hline Schizoaffective disorder & 178 & 9.1 \\
\hline Bipolar disorder & 255 & 13.1 \\
\hline Depressive disorder & 781 & 40.1 \\
\hline Anxiety disorder & 82 & 4.2 \\
\hline Psychosis, not other specified (PNOS) & 227 & 11.6 \\
\hline Other minor psychiatric disorders & 212 & 10.9 \\
\hline
\end{tabular}

Note: *valid case only.

Table 2. Patterns of substance use $(n=1949)$.

\begin{tabular}{ccc}
\hline Variable & $\boldsymbol{n}$ & $\%$ \\
\hline Types of substance & 1204 & \\
Alcohol & 1000 & 61.8 \\
Stimulants & 626 & 51.3 \\
Marijuana & 358 & 32.1 \\
Opiate & 175 & 18.4 \\
Sedatives/Hypnotics & 125 & 9.0 \\
Hallucinogens & & 6.4 \\
Types of substance user & 1042 & \\
Polysubstance user & 907 & 53.5 \\
Single/No substance user & & 46.5 \\
\hline
\end{tabular}


Table 3. Chi-square analyses for physical disease between polysubstance and single/no substance users $(n=1949)$.

\begin{tabular}{|c|c|c|c|c|c|c|}
\hline \multirow[t]{2}{*}{ Variable } & \multicolumn{2}{|c|}{ Polysubstance user } & \multicolumn{2}{|c|}{$\begin{array}{c}\text { Single/No } \\
\text { substance user }\end{array}$} & \multirow[t]{2}{*}{$\chi^{2}(p)$} & \multirow[t]{2}{*}{ Total (\%) } \\
\hline & $n$ & $\%$ & $n$ & $\%$ & & \\
\hline Circulatory Disease & 224 & 48.8 & 235 & 51.2 & $1.7(0.19)$ & $459(24.0)$ \\
\hline Respiratory Disease & 174 & 57.0 & 131 & 43.0 & $5.0^{*}$ & $305(15.6)$ \\
\hline Digestive Disease & 265 & 59.6 & 180 & 40.4 & $8.3^{* *}$ & $445(22.8)$ \\
\hline Endocrine Disease & 100 & 45.2 & 121 & 54.8 & $6.4^{*}$ & $221(11.3)$ \\
\hline Musculoskeletal Disease & 222 & 61.0 & 142 & 39.0 & $9.8^{* *}$ & $364(17.4)$ \\
\hline HIV/AIDS infection & 92 & 66.7 & 46 & 33.3 & $9.8^{\star *}$ & $138(7.1)$ \\
\hline
\end{tabular}

Note: ${ }^{*} p<0.05 ;{ }^{* *} p<0.01$.

disease ( $n=305,15.6 \%)$, endocrine disease ( $n=221,11.3 \%)$, and HIV/AIDS infection $(n=138,7.1 \%)$. Chi-square results showed that polysubstance users reported significantly higher proportions of respiratory disease $(57.0 \%$ vs. $43.0 \%, p$ $<0.05$ ), digestive disease (59.6\% vs. $40.4 \%, p<0.01$ ), musculoskeletal disease (61.0\% vs. $39.0 \%, p<0.01)$, and HIV/AIDS infection ( $66.7 \%$ vs. $33.3 \%, p<0.01)$ compared to single/no substance users. On the contrary, polysubstance users reported a significantly lower proportion of endocrine disease ( $45.2 \%$ vs. $54.8 \%$, $p<0.05)$ compared to single/no substance users. There was no significant difference in the prevalence of circulatory disease between the two groups.

\section{Discussion}

This study investigated the prevalence of co-occurring polysubstance use and whether there was a significant difference in physical diseases between polysubstance users and single/no substance users in residential treatment programs. The prevalence of co-occurring polysubstance use was $53.5 \%$ in this study population. Chi-square analyses revealed that polysubstance users with any type of mental disorder reported more respiratory disease, digestive disease, musculoskeletal disease, and HIV/AIDS infection but less endocrine disease than single/no substance users.

Over $60 \%$ of this study population was homeless due to the characteristics of the study settings, residential treatment programs located in the San Francisco Bay area, which has a high number of homeless people. The population also showed a higher prevalence of polysubstance use which is consistent with findings from another study showing that an unstable housing condition, especially homelessness, is a risk factor for substance use [11]. In this study, depressive disorder was a major psychiatric disorder which was inconsistent with the findings from another study with Asian Americans in which more than half of the study sample had schizophrenia and schizoaffective disorder [12]. This suggests that further analyses are recommended to examine racial/ethnic differences in the prevalence of psychiatric disorders. Schizospectrum disorders including schi- 
zophrenia, schizoaffective disorder, and PNOS took the $2^{\text {nd }}$ major psychiatric disorder. Bipolar disorder and anxiety disorder, however, were uncommon in this study sample. Although there are psychiatric diagnoses differences in the prevalence of substance use, i.e. depressive disorder is a risk factor for alcohol use whereas schizophrenia is a protective factor for alcohol use [12] [13] [14], having a mental disorder is generally considered to increase vulnerability to substance use. When considering that $40 \%$ of this study sample had depressive disorder, it is not surprising that the prevalence of co-occurring polysubstance use was $53.5 \%$ in this study population, which was comparable to findings from SAMHSA [7]. Combination patterns of substances have not been examined in this study, however, other literatures consistently have supported that the combination of alcohol and one or more substances is the common polysubstance pattern [8] [15].

Findings from this study identified that polysubstance users with mental disorders experienced more digestive and HIV/AIDS diseases, which were consistent with findings from another study that showed co-occurring substance use as a risk factor for digestive and infectious diseases [5]. Moreover, this study found that polysubstance users reported more respiratory and musculoskeletal diseases because using multiple substances rather than a single substance may have more deteriorating effects on almost all body systems. On the other hand, polysubstance users in this study reported less endocrine disease which was consistent with report from Frasch et al's study [5]. This finding can be supported by the understanding that endocrine disease, especially diabetes, is a common complication of $2^{\text {nd }}$ generation antipsychotics which are often used to treat psychotic symptoms for schizophrenic patients, and polysubstance use is less common among schizophrenic patients [13] [14]. Further studies are needed to examine the interactions between polysubstance use and each psychiatric disorder on each physical disease to specifically determine which patients are at high risk.

Dickey and colleagues [4] found that the total mean annual treatment cost for schizophrenic patients with substance use disorder was $75 \%$ higher than that of schizophrenic patients without substance use disorder and that the number of people hospitalized was also three times higher in the comorbidity group. However, there is a lack of studies comparing treatment costs and hospitalization rates between co-occurring polysubstance users and single/no substance users receiving care for their physical diseases. Integrated treatment services for patients with psychiatric disorders and co-occurring substance use along with prevention and early screening strategies should be expanded to improve physical health outcomes, thereby reducing medical costs.

\section{Study Limitations}

This study used a cross-sectional design to explore differences in the prevalence of physical disease by polysubstance users and single/no substance users with mental disorders in residential treatment programs, meaning a causal relation- 
ship cannot be guaranteed. The sample for this study came from residential treatment programs where the homeless were overrepresented; therefore, there may be skewed findings such as the high prevalence of severe mental disorders, polysubstance use, and physical diseases. Due to the limitations of the Chi-square analyses used in this study, important variables such as age, smoking status, and mental disorders which may be potential risk factors for physical disease, were not controlled, indicating the need for further research study.

\section{Conclusion}

This study found that polysubstance users with mental disorders had more respiratory, digestive, musculoskeletal, and HIV/AIDS infectious diseases compared to single/no substance users with mental disorders. Therefore, strategies to manage comorbid physical disease and polysubstance use in patients with mental disorders should include prevention and early screening of co-occurring polysubstance use in high-risk groups, and the development and implementation of appropriate and adequate comprehensive treatment services to integrate medical and psychiatric services.

\section{Acknowledgements}

The authors would like to acknowledge Michelle Grace Lee, graduate of the School of Nursing, San Diego State University, for her manuscript editing.

\section{Funding}

This study was not funded by any institution or body.

\section{Conflicts of Interest}

The authors declare that they have no competing interests.

\section{References}

[1] Substance Abuse and Mental Health Services Administration (2019) Key Substance Use and Mental Health Indicators in the United States: Results from the $2018 \mathrm{Na}$ tional Survey on Drug Use and Health. HHS Publication No. PEP19-5068, NSDUH Series H-54, Center for Behavioral Health Statistics and Quality, Substance Abuse and Mental Health Services Administration, Rockville.

https://www.samhsa.gov/data/

[2] Mericle, A.A., Ta Park, Van M., Holck, P. and Arria, A.M. (2012) Prevalence, Patterns, and Correlates of Co-Occurring Substance Use and Mental Disorders in the United States: Variations by Race/Ethnicity. Comprehensive Psychiatry, 53, 657-665. https://doi.org/10.1016/j.comppsych.2011.10.002

[3] Smith, M.J., Thirthalli, J., Ben Abdallah, A., Murray, R.M. and Cottler, L.B. (2009) Prevalence of Psychotic Symptoms in Substance Users: A Comparison across Substances. Comprehensive Psychiatry, 50, 245-250. https://doi.org/10.1016/j.comppsych.2008.07.009

[4] Dickey, B., Normand, S.-L.T., Weiss, R.D., Drake, R.E. and Azeni, H. (2002) Medical Morbidity, Mental Illness, and Substance Use Disorders. Psychiatric Services, 53, 
861-867. https://doi.org/10.1176/appi.ps.53.7.861

[5] Frasch, K., Ivar Larsen, J., Cordes, J., Jacobsen, B., Olrik Wallenstein Jensen, S., Lauber, C., et al. (2012) Physical Illness in Psychiatric Inpatients: Comparison of Patients with and without Substance Use Disorders. International Journal of Social Psychiatry, 59, 757-764. https://doi.org/10.1177/0020764012456803

[6] Smith, G.W., Farrell, M., Bunting, B.P., Houston, J.E. and Shevlin, M. (2011) Patterns of Polydrug Use in Great Britain: Findings from a National Household Population Survey. Drug and Alcohol Dependence, 113, 222-228.

https://doi.org/10.1016/j.drugalcdep.2010.08.010

[7] Substance Abuse and Mental Health Services Administration (2006) Results from the 2005 National Survey on Drug Use and Health: National Findings. Office of Applied Studies, NSDUH Series H-30, DHHS Publication No. SMA 06-4194, Substance Abuse and Mental Health Services Administration, Rockville.

[8] Kedia, S., Sell, M.A. and Relyea, G. (2007) Mono- versus Polydrug Abuse Patterns among Publicly Funded Clients. Substance Abuse Treatment, Prevention, and Policy, 2, Article No. 33. https://doi.org/10.1186/1747-597X-2-33

[9] American Psychiatric Association (1994) Diagnostic and Statistical Manual of Mental Disorders. Fourth Edition (DSM-IV), American Psychiatric Association, Washington DC.

[10] World Health Organization (1998) International Classification of Diseases, Ninth Revision (ICD-9). http://ftp.cdc.gov/pub/Health_Statistics/NCHS/Publications/ICD-9

[11] Palepu, A., Patterson, M., Strehlau, V., Moniruzzamen, A, Tan de Bibiana, J., Frankish, J., et al. (2012) Daily Substance Use and Mental Health Symptoms among a Cohort of Homeless Adults in Vancouver, British Columbia. Journal of Urban Health, 90, 740-746. https://doi.org/10.1007/s11524-012-9775-6

[12] Kim, M., Lee, J., Nakajima, M. and Chafetz, L. (2019) Predictors of Co-Occurring Substance Use among Asian Americans in Residential Treatment Programs. Open Journal of Psychiatry, 9, 153-164. https://doi.org/10.4236/ojpsych.2019.92012

[13] Chafetz, L., White, M.C., Collins-Bride, G. and Nickens, J. (2005) The Poor General Health of the Severely Mentally Ill: Impact of Schizophrenic Diagnosis. Community Mental Health Journal, 41, 169-184. https://doi.org/10.1007/s10597-005-2651-z

[14] Chafetz, L., White, M.C., Collins-Bride, G., Nickens, J. and Cooper, B.A. (2006) Predictors of Physical Functioning among Adults with Severe Mental Illness. Psychiatric Services, 57, 225-231. https://doi.org/10.1176/appi.ps.57.2.225

[15] Liu, Y.Y., Elliott, A.L., Serdarevic, M., Leeman, R.F. and Cottler, L.B. (2019) A Latent Class Analysis of the Past-30-Day Substance Use Patterns among Lifetime Cocaine Users: Findings from a Community Sample in North Central Florida. Addictive Behaviors Reports, 9, Article ID: 100170.

https://doi.org/10.1016/j.abrep.2019.100170 